\title{
La diferencia entre tiempo programado y efectivo en hemodiafiltración en línea: ¿Cada minuto cuenta en la eficacia dialítica?
}

\author{
Ana Vanessa Fernández Martínez, Rocío Pérez Díaz, Laura Pérez Valencia, Virginia Caparrós Ríos, \\ María José Espinosa Sáez, Verena Romero Willhoft, María Soledad Pereira Martínez
}

Enfermeras. Centros de diálisis NephroCare. Fresenius Medical Care Services

Cartagena y San Pedro del Pinatar. Murcia

\section{Resumen}

\section{Introducción}

El factor tiempo es determinante en la eficacia de la diálisis, recomendando las European Best Practices Guides of Dialysys Adequaccy una duración semanal no inferior a 720 minutos.

Los modernos monitores de diálisis interrumpen el procedimiento por auto chequeos que incrementan la seguridad. Esos minutos de diferencia no han sido tenidos en cuenta en otros resultados obtenidos, algún autor ya hace referencia a la necesidad de contemplarlos.

Los objetivos del presente estudio fueron determinar la diferencia real entre el tiempo programado y el tiempo efectivo de diálisis, y una vez comprobado incrementar la duración de la sesión para hacer que el tiempo efectivo sea realmente el deseado en cada paciente, y nunca inferior a 240 minutos por sesión. Finalmente comprobamos si existen diferencias en la eficacia dialítica, midiendo el Kt y el volumen total de reinfusión.

\section{Pacientes y métodos}

Diseñamos un estudio prospectivo sobre población prevalente en hemodiafiltración on-line. Reclutamos

Correspondencia:

Ana Vanessa Fernández Matínez

Centro de diálisis NephroCare

Paseo Alfonso XIII N61

30202. Cartagena. Murcia

e-mail: nefroclubcarthago@gmail.com
152 pacientes en hemodiafiltración on-line. Durante 6 sesiones consecutivas ( 912 sesiones) la diálisis se efectuó con un tiempo programado $\geq 240$ minutos. En un segundo periodo 6 sesiones consecutivas ( 912 sesiones), la sesión de diálisis se alargó hasta un tiempo efectivo igual al previo programado.

La variable principal en estudio fue el tiempo, siendo variables secundarias el flujo sanguíneo, $\mathrm{Kt}$, y volumen total de reinfusión. En el segundo periodo se recogieron posibles complicaciones intradialíticas en el tiempo añadido.

\section{Resultados}

Se analizan 152 pacientes, 66\% hombres, de 65,7 $\pm 14,9$ (26-88) años de edad, con una permanencia en diálisis de $56,5 \pm 59,5$ meses, en un total de 1824 sesiones de hemodiafiltración on-line.

No se aprecian diferencias significativas en $Q b$ $(428,74 \pm 39,73$ vs $429,86 \pm 38,54 \mathrm{ml} / \mathrm{min})$.

El tiempo medio de la sesión aumenta significativamente $(p<0,001)$ en 7 minutos (de $233,58 \pm 3,29$ hasta $240,67 \pm 2,71$ minutos).

El Kt aumenta significativamente $(p<0,001)$ un $3,2 \%$ (desde $60,06 \pm 5,86$ hasta $61,99 \pm 5,80$ litros), disminuyendo el \% de sesiones en las que no se alcanza el Kt deseado por superficie corporal (de $5,7 \%$ a $3,1 \%$ ).

El volumen total de reinfusión se incrementa significativamente $(p<0,001$ ) un $1,5 \%$ (de $24,10 \pm 2,72$ a $24,46 \pm 2,77$ litros), con un $\%$ similar de sesiones donde no se alcanzan los 20 litros objetivo $(4,6 \%$ versus $4,3 \%$ ). 
No se observan complicaciones intradialíticas en el periodo de tiempo incrementado ni episodios de coagulación.

\section{Discusión y conclusiones}

Tanto Kt como volumen total de reinfusión son los principales indicadores reconocidos para medir la adecuación de la diálisis en la hemodiafiltración on-line.

El Kt ha sido referido por algunos autores como un indicador de dosis de diálisis más exigente que el $\mathrm{Kt} / \mathrm{V}$. El tiempo efectivo de la sesión es un factor clave para la consecución del Kt óptimo ajustado a superficie corporal y objetivos de volumen total de reinfusión en el paciente en hemodiafiltración online posdilucional, con importantes implicaciones en la mortalidad.

En el tratamiento con hemodiafiltración on-line posdilucional todos los minutos son importantes. Se debe considerar el incremento del tiempo programado (aproximadamente 7 minutos por sesión) para conseguir que el tiempo efectivo sea igual o superior a 720 minutos/semana, consiguiendo optimizar tanto la dosis de diálisis como el volumen de reinfusión.

\section{PALABRAS CLAVE: \\ - TIEMPO EFECTIVO \\ - TIEMPO PROGRAMADO \\ - EFICACIA DIALÍTICA}

The difference between programmed time and effective time in online haemodiafiltration: Does every minute count in dialytic efficiency?

\section{Abstract}

\section{Aim}

To determine the difference between programmed time and effective dialysis time, and once it has been found, to increase the duration of the session so that the effective time is actually as desired for each patient, and never less than 240 minutes per session. Finally, we determined whether there are differences in dialytic efficiency, by measuring $\mathrm{Kt}$ and total reinfusion volume.

\section{Patients and methods}

We designed a prospective study on the prevalent population in online haemodiafiltration. We recruited 152 patients undergoing online haemodiafiltration. For 6 consecutive sessions ( 912 sessions), the dialysis was carried out with a programmed time $\geq 240$ minutes. In a second period of 6 consecutive sessions ( 912 sessions), the dialysis session was extended to an effective time equal to the previously programmed time.

The principal variable in the study was time, with blood flow, Kt and total reinfusion volume as secondary variables. In the second period, any intradialytic complications in the added time were noted.

\section{Results}

A total of 152 patients were analysed, $66 \%$ of whom were man, $65.7 \pm 14.9$ (26-88) years old, with 56.5 \pm 59.5 months on dialysis, in a total of 1824 online haemodiafiltration sessions.

No significant differences in $\mathrm{Qb}$ were observed $(428.74 \pm 39.73$ v. $429.86 \pm 38.54 \mathrm{ml} / \mathrm{min})$.

The average duration of the session increased significantly $(p<0.001)$ by 7 minutes (from $233.58 \pm 3.29$ to $240.67 \pm 2.71$ minutes).

Kt increased significantly $(p<0.001$ ) by $3.2 \%$ (from $60.06 \pm 5.86$ to $61.99 \pm 5.80$ litres), with a reduction in the \% of sessions in which the desired Kt per body surface area is not reached (from $5.7 \%$ to $3.1 \%$ ). The total reinfusion volume increased significantly $(p<0.001$ ) by $1.5 \%$ (from $24.10 \pm 2.72$ to $24.46 \pm 2.77$ litres), with a similar \% of sessions in which the target of 20 litres was not reached (4.6\% compared to $4.3 \%$ ).

No intradialytic complications or coagulation episodes were observed in the increased time period.

\section{Discussion and conclusions}

Both $\mathrm{Kt}$ and total reinfusion volume are the main acknowledged indicators for measuring the adequacy of dialysis in online haemodiafiltration.

Kt has been referred to by some authors as a more demanding dialysis does indicator than $\mathrm{Kt} / \mathrm{V}$. The effective time of the session is a key factor for achieving optimal Kt tailored to body surface area 
and total reinfusion volume targets in post-dilutional online haemodiafiltration patients, with important implications for mortality.

In post-dilutional online haemodiafiltration, every minute is important. Increasing the programmed time (by approximately 7 minutes per session) should be considered so that the effective time is equal to or greater than 720 minutes/week, managing to optimize both the dialysis dose and the reinfusion volume.

\section{KEY WORDS}

- EFFECTIVE TIME

- PROGRAMMED TIME

- DIALYTIC EFFICIENCY

\section{Introducción}

El factor tiempo es determinante en la eficacia de la diálisis, siendo recomendada por las Eurepean Best Practices Guides of Dialysys Adequaccy ${ }^{1}$ una duración semanal no inferior a 720 minutos en pacientes con tres sesiones semanales. Esa duración puede verse incrementada en situaciones de hipertensión arterial refractaria pese a la máxima ultrafiltración, inestabilidad cardiovascular o hemodinámica, hiperfosforemia resistente a quelantes y malnutrición.

Sin embargo, los modernos monitores de diálisis interrumpen el procedimiento por auto chequeos que incrementan la seguridad comprobando la permeabilidad del sistema hidraúlico (test cíclicos de presión). Aparentemente, esos minutos de diferencia no han sido tenidos en cuenta en los resultados obtenidos, si bien algún autor ya hace referencia a la necesidad de contemplarlos ${ }^{3}$.

Los objetivos del presente estudio fueron determinar la diferencia real entre el tiempo programado y el tiempo efectivo de diálisis, y una vez comprobado incrementar la duración de la sesión para hacer que el tiempo efectivo sea realmente el deseado en cada paciente, y nunca inferior a 240 minutos por sesión.
Finalmente comprobamos si existen diferencias en la eficacia dialítica, midiendo el Kt y el volumen total de reinfusión (VTR).

\section{Pacientes y métodos}

Diseñamos un estudio prospectivo sobre población prevalente en hemodiafiltración on-line en dos centros de diálisis. Todos los pacientes dan su consentimiento expreso a participar en este estudio. Reclutamos 152 pacientes en hemodiafiltración on-line. Durante 6 sesiones consecutivas (912 sesiones) la diálisis se efectuó con un tiempo programado $\geq 240$ minutos. En un segundo periodo de 6 sesiones consecutivas (912 sesiones), la sesión de diálisis se alargó hasta alcanzar un tiempo efectivo igual al previo programado, sin modificaciones en el resto de la programación de la pauta de diálisis.

Todas las sesiones de diálisis se realizaron con el Sistema terapéutico 5008 (FMC), que determina el tiempo programado y el tiempo efectivo de cada sesión.

La variable principal en estudio fue el tiempo, siendo variables secundarias el flujo sanguíneo $(\mathrm{Qb}), \mathrm{Kt}$, y VTR. En el segundo periodo se recogieron posibles complicaciones intradialíticas en el tiempo añadido, así como posibles coagulaciones del sistema extracorpóreo, dado que pese al aumento de tiempo, no se modificaron las dosis de heparina.

Para el análisis estadístico se utilizó el programa SPSS 13.0 para Windows. Las variables cuantitativas se mostraron como media y desviación estándar. Las variables cualitativas como frecuencias y porcentajes. EI contraste de hipótesis se realizó mediante la T-student para muestras relacionadas en las variables cuantitativas, y la chi-cuadrado en las variables cualitativas.

\section{Resultados}

Se analizan 152 pacientes, 66\% hombres, de 65,7 $\pm 14,9$ (26-88) años de edad, con una permanencia en diálisis de $56,5 \pm 59,5$ meses (3-324) y etiologías conocidas más frecuentes hipertensiva $(22,4 \%)$ y diabética $(22,4 \%)$ en un total de 1824 sesiones de hemodiafiltración on-line. 
Según se aprecia en la figura 1 , no existen diferencias significativas en $Q b(428,74 \pm 39,73$ vs $429,86 \pm 38,54$ $\mathrm{ml} / \mathrm{min}$ ).

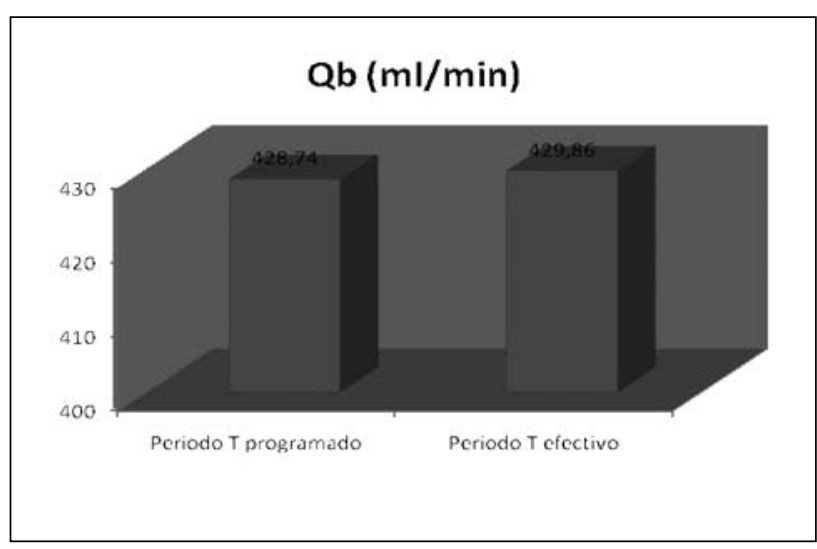

Figura 1. Resultados de Qb

El tiempo medio de la sesión aumenta significativamente $(p<0,001$ ) en 7 minutos (de 233,58 $\pm 3,29$ hasta $240,67 \pm 2,71$ minutos), tal como se aprecia en la figura 2.

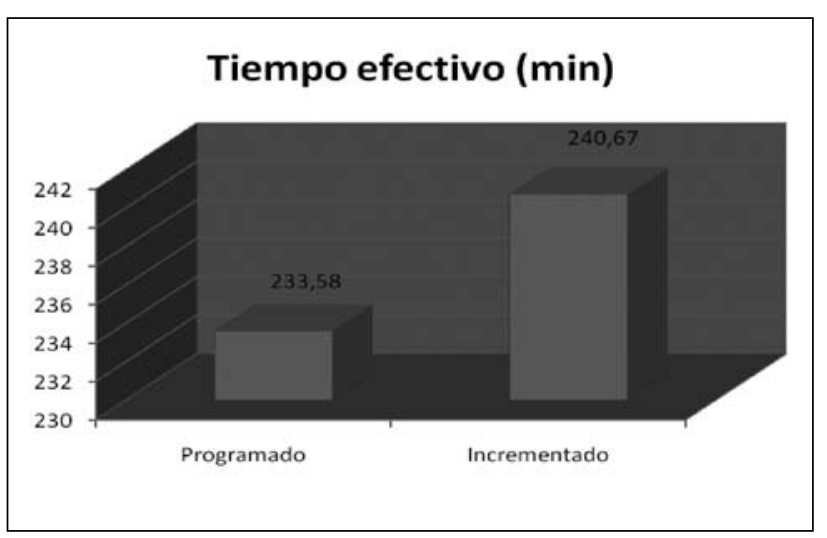

Figura 2. Resultados de Tiempo

El Kt (figura 3) aumenta significativamente $(p<0,001)$ un $3,2 \%$ (desde $60,06 \pm 5,86$ hasta $61,99 \pm 5,80$ litros), disminuyendo el \% de sesiones en las que no se alcanza el Kt deseado por superficie corporal (de $5,7 \%$ a $3,1 \%$ ).

EI VTR (Figura 4) se incrementa significativamente $(p<0,001)$ un $1,5 \%$ (de $24,10 \pm 2,72$ a $24,46 \pm 2,77$ litros), con un \% similar de sesiones donde no se alcanzan los 20 litros objetivo (4,6\% versus $4,3 \%$ ).

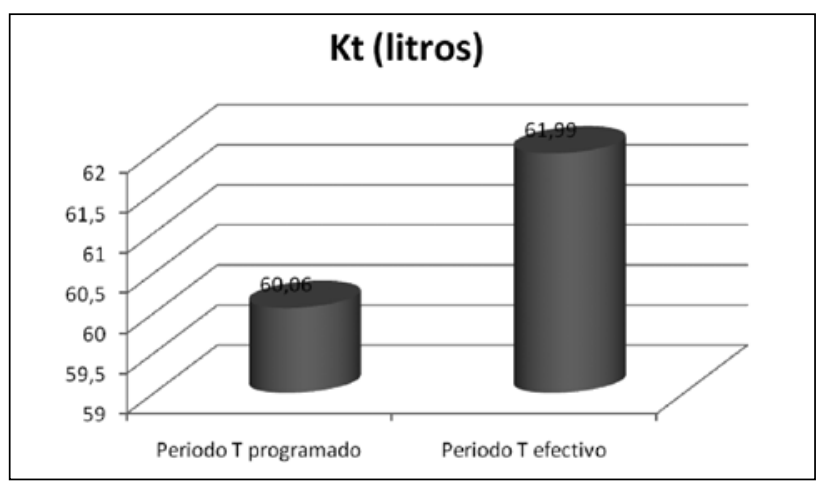

Figura 3. Resultados de Kt

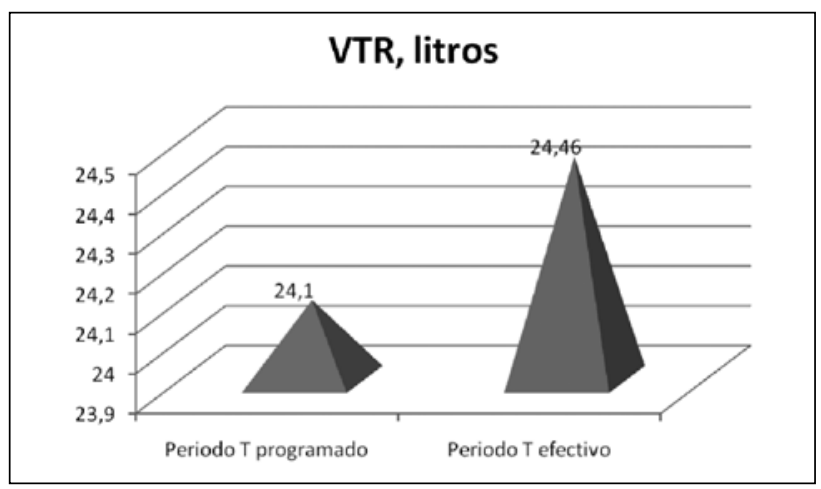

Figura 4. Resultados de VTR

No se observan complicaciones intradialíticas en el periodo de tiempo incrementado ni episodios de coagulación.

\section{Discusión}

Tanto Kt como VTR son los principales indicadores reconocidos para medir la adecuación de la diálisis en la hemodiafiltración on-line ${ }^{4}$.

El Kt ha sido referido por algunos autores ${ }^{5,6}$ como un indicador de dosis de diálisis más exigente que el Kt/V puesto que nos permite discriminar mejor los casos de infra diálisis. El tiempo efectivo de la sesión es un factor clave para la consecución del Kt óptimo ajustado a superficie corporal ${ }^{7}$, con importantes implicaciones en la mortalidad ${ }^{8}$.

Por otro lado, el tiempo efectivo de la sesión también es un factor esencial para la consecución de los objetivos de volumen total de reinfusión en el paciente en hemodiafiltración on-line posdilucional, tanto en su forma automatizada ${ }^{9}$ como en la automatizada manual ${ }^{10}$. 
Altos volúmenes convectivos condicionan la bondad de los resultados de esta técnica, incluso en lo referente a la reducción de la mortalidad ${ }^{11-13}$.

Los resultados de nuestro estudio confirman como la implicación enfermera en el cumplimiento del tiempo programado como tiempo efectivo incrementan la eficacia de la diálisis, un 3,2\% en la dosis medida por Kt y un $1,5 \%$ en el volumen de reinfusión, sin que se hayan producido efectos adversos en ese tiempo extendido.

\section{Conclusión}

En el tratamiento con hemodiafiltración on-line posdilucional todos los minutos son importantes. Se debe considerar el incremento del tiempo programado (aproximadamente 7 minutos por sesión) para conseguir que el tiempo efectivo sea igual o superior a 720 minutos/semana, consiguiendo optimizar tanto la dosis de diálisis ( $3 \%$ ) como el volumen de reinfusión ( $1,5 \%$ ) en pacientes en hemodiafiltración en línea. Para ello, la actuación enfermera es fundamental.

Recibido: 20 septiembre 2012

Revisado: 24 0ctubre 2012

Modificado: 5 Noviembre 2012

Aceptado: 6 Noviembre 2012

\section{Bibliografía}

1. Tattersall J, Martin-Malo A, Pedrini L, y cols. EBPG guideline on dialysis strategies. Nephrol Dial Transplant (2007) 22 [Suppl 2]: ii5-ii21.

2. Guía Sistema Terapéutico 5008. Fresenius Medical Care.

3. Molina M, Roca S, De Alarcón RM, y cols. Cálculo del Kt como indicador de calidad en el área de adecuación en hemodiálisis. Nefrología 2010: 30 (3): 215-221.

4. Fernández $A V$, Piñero J, Arregui Y y cols. ¿Qué indicadores son considerados por enfermería para conseguir una diálisis perfecta en el paciente en hemodiafiltración on-line? Comunicación presenta- da en el XXXVI Congreso de la Sociedad Española de Enfermería Nefrológica. Sevilla, 2011.

5. Maduell F, Vera M, Serra N, y cols. Kt como control y seguimiento de la dosis en una unidad de hemodiálisis. Nefrología 2008; 28 (1): 43-47.

6. Fernández $A V$, Soto $S$, Arenas $M$ y cols Estudio comparativo de la dosis de dialisis medida por (Kt) y KtV. 21 Rev Soc Esp Enferm Nefrol 2009; 12(2): 97-102.

7. Fernández AV, Pereira MS, Vilar MV y cols. Kt como indicador de dosis adecuada en una unidad de hemodiálisis: Estudio prospectivo. Libro de comunicaciones presentadas al XXXIV Congreso Nacional de la SEDEN. Pamplona 2009.

8. Lowrie EG, Li Z, Ofhsum NJ and Lazarus JM. Evaluating a new method to judge dialysis treatment using online measurements of ionics clearance. Kidney Int 2006; 60: 211-217.

9. Fernández $\mathrm{AV}$, Soto $\mathrm{S}$, Arenas $\mathrm{M}$ y cols. Comparación de infusión automática respecto a manual en hemodiafiltracion on line postdilucional. $17 \mathrm{Rev}$ Soc Esp Enferm Nefrol 2010; 13 (1):17-22.

10. Fernández $A V$, Horrillo $F$, Pérez Valencia $L$, y cols. Infusión automatizada manual en la hemodiafiltración on-line posdilucional: hacia la optimización del volumen de reinfusión. Rev Soc Esp Enferm Nefrol 2011; 14 (3): 35-39.

11. Canaud B, Bragg-Gresham JL, Marshall JR, y cols. Mortality risk for patients receiving hemodiafiltration versus hemodialysis: European results from the DOPPS. Kidney Int 2006;69:2087-93.

12. $0 k E, A s c i G, 0 k E S$, y cols. Comparission of posdilution on-line hemodiafiltration and hemodialysis (TURKEY HDF STUDY). Personal communication presented in the EDTA Congress (LBCT2), Prague, 2011.

13. Grooteman $M$, Van der Dorpel $R$, Boots $M$, y cols. On-line hemodiafiltration versus low-flux hemodialysis. Effects on all-cause mortality and cardiovascular events in a randomized controlled trial: The convective transport study (CONTRAST). Personal communication presented in the EDTA Congress (LBCT3), Prague, 2011. 